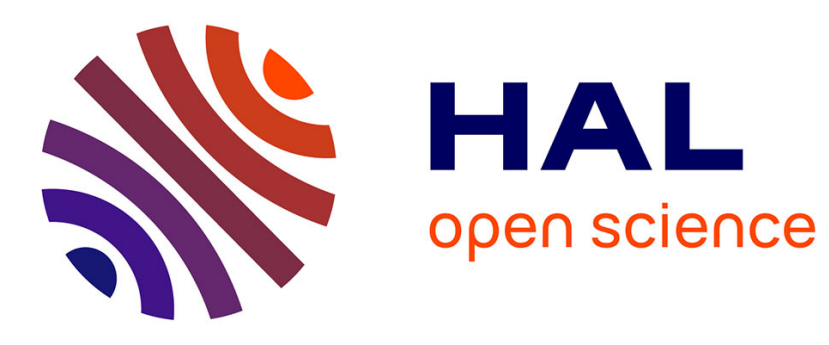

\title{
Core-shell-like Au sub-nanometer clusters in Er-implanted silica
}

\author{
Chiara Maurizio, Tiziana Cesca, Giovanni Perotto, Boris Kalinic, Niccolò \\ Michieli, Carlo Scian, Yves Joly, Giancarlo Battaglin, Paolo Mazzoldi, \\ Giovanni Mattei
}

\section{To cite this version:}

Chiara Maurizio, Tiziana Cesca, Giovanni Perotto, Boris Kalinic, Niccolò Michieli, et al.. Coreshell-like Au sub-nanometer clusters in Er-implanted silica. Nanoscale, 2015, 7 (19), pp.8968. 10.1039/c5nr01564b . hal-01151249

\author{
HAL Id: hal-01151249 \\ https://hal.science/hal-01151249
}

Submitted on 12 May 2015

HAL is a multi-disciplinary open access archive for the deposit and dissemination of scientific research documents, whether they are published or not. The documents may come from teaching and research institutions in France or abroad, or from public or private research centers.
L'archive ouverte pluridisciplinaire HAL, est destinée au dépôt et à la diffusion de documents scientifiques de niveau recherche, publiés ou non, émanant des établissements d'enseignement et de recherche français ou étrangers, des laboratoires publics ou privés. 


\section{Nanoscale}

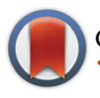

CrossMark \& click for updates

Cite this: Nanoscale, 2015, 7, 8968

Received 10th March 2015, Accepted 3rd April 2015

DOI: 10.1039/c5nr01564b

www.rsc.org/nanoscale

\section{Core-shell-like Au sub-nanometer clusters in Er-implanted silica}

\author{
Chiara Maurizio, ${ }^{* a}$ Tiziana Cesca, ${ }^{a}$ Giovanni Perotto, $\dagger^{a}$ Boris Kalinic, ${ }^{a}$ \\ Niccolò Michieli, ${ }^{a}$ Carlo Scian, ${ }^{a}$ Yves Joly, ${ }^{b, c}$ Giancarlo Battaglin, ${ }^{d}$ Paolo Mazzoldi ${ }^{a}$ \\ and Giovanni Mattei ${ }^{\mathrm{a}}$
}

\begin{abstract}
The very early steps of Au metal cluster formation in Er-doped silica have been investigated by highenergy resolution fluorescence-detected X-ray absorption spectroscopy (HERFD-XAS). A combined analysis of the near-edge and extended part of the experimental spectra shows that Au cluster nucleation starts from a few $\mathrm{Au}$ and $\mathrm{O}$ atoms covalently interconnected, likely in the presence of embryonic $\mathrm{Au}-\mathrm{Au}$ correlation. The first Au clusters, characterized by a well defined Au-Au coordination distance, form upon $400{ }^{\circ} \mathrm{C}$ inert annealing. The estimated upper limit of the Gibbs free energy for the associated heterogeneous nucleation is $0.06 \mathrm{eV}$ per atom, suggesting that the Au nucleation is assisted by matrix defects, most likely non-bridging oxygen atoms. The experimental results indicate that the formed subnanometer Au clusters can be applied as effective core-shell systems in which the Au atoms of the 'core' develop a metallic character, whereas the Au atoms in the 'shell' can retain a partially covalent bond with $O$ atoms of the silica matrix. High structural disorder at the Au site is found upon neutral annealing at a moderate temperature $\left(600^{\circ} \mathrm{C}\right)$, likely driven by the configurational disorder of the defective silica matrix. A suitable choice of the Au concentration and annealing temperature allows tailoring of the Au cluster size in the sub-nanometer range. The interaction of the Au cluster surface with the surrounding silica matrix is likely responsible for the infrared luminescence previously reported on the same systems.
\end{abstract}

\section{Introduction}

Noble metal nanoparticles have found many technological applications in different fields, such as catalysis, ${ }^{1-3}$ sensing,,${ }^{4,5}$ biomedicine $^{6}$ and photovoltaics. ${ }^{7}$ Because of the size dependence of their peculiar optical, electronic and catalytic properties, ${ }^{8-10}$ a strong effort is currently being made to achieve precise control of the cluster size and number density. In a bottom-up approach, good control of the aggregate size distribution is possible only if the whole process of cluster nucleation and growth is deeply understood. The first steps of cluster aggregation are very interesting not only to achieve full control of the cluster size in the nanometer range, but also per se in the sense that the electronic energy levels of few-atom clusters do not exhibit a metallic character and are in principle

\footnotetext{
${ }^{a}$ Physics and Astronomy Department and CNISM, University of Padova, via Marzolo 8, I-35131 Padova, Italy. E-mail: chiara.maurizio@unipd.it

${ }^{b}$ Univ. Grenoble Alpes, Inst NEEL, F-38042 Grenoble, France

${ }^{c}$ CNRS, Inst NEEL, F-38042 Grenoble, France

${ }^{d}$ Department of Molecular Sciences and Nanosystems, Ca' Foscari University, Dorsoduro 2137, I-30123 Venezia, Italy

$\dagger$ Present address: Biomedical Engineering Department, Tufts University, 4, Colby Street, 02155 Medford, MA.
}

size dependent, resulting in interesting optical emission properties. ${ }^{10,11}$

These considerations have motivated numerous experimental investigations on metal nanocluster formation, especially in liquid media, upon different chemical processes. ${ }^{12-14}$ The reported experiments monitor the metal nucleation upon chemical reduction and aggregation, but structural analyses generally skip the nucleation process itself, where aggregates of size critical for the thermodynamic stability are made of a few atoms. Metal nucleation in glass or solid matrices in general is particularly interesting, since these composite materials are prototypical systems for optoelectronic and photovoltaic applications. They also offer the advantage that diffusion-induced phenomena occur on a much longer time scale, so that they can more easily be tracked. Most of the experiments on cluster nucleation in solids rely on the optical properties of ions and multimers, ${ }^{15,16}$ while a detailed structural investigation is often missing.

X-ray absorption spectroscopy (XAS) performed at the metal edge is a suitable technique to monitor metal nucleation and the first steps of growth since it can detect both oxide phases ${ }^{17}$ and the formation of clusters, ${ }^{14,18}$ even in the sub-nanometer range of size. ${ }^{19,20}$ In the case of monodisperse clusters, a detailed analysis can also give an indication of the cluster 
shape. ${ }^{21,22}$ By correlating XAS with photoluminescence spectroscopy, we have recently shown that clusters of a few $\mathrm{Au}$ atoms in silica absorb energy in the visible-UV range and can efficiently transfer part of it to nearby $\mathrm{Er}^{3+}$ ions, dramatically increasing the rare earth effective excitation cross-section, with promising photonic applications. ${ }^{19,23}$ This energy transfer process has been related to electronic surface states of molecule-like clusters resonant with the corresponding $\mathrm{Er}^{3+}$ absorption level, ${ }^{11}$ indicating a critical role of the cluster-matrix interface in this process. Nevertheless, at present experimental results on this interesting aspect are missing.

In this work we have examined the Au cluster formation in Er-doped silica prepared by ion implantation and followed by suitable annealing under specific atmospheres. This matrix has been chosen in view of its application in actual optoelectronic devices like Er-doped fiber amplifiers. To gain insight into the cluster-matrix interface and the very first steps of cluster nucleation, the Au concentration has been lowered by more than one order of magnitude with respect to our previous work, ${ }^{19}$ and a new XAS-based technique has been employed. In particular, the Au local concentration has been varied over more than one order of magnitude: this approach, coupled with different specific annealing conditions, allowed preparation of systems with different and defined cluster sizes, always in the sub-nanometer range. Advanced high-energyresolution fluorescence-detected X-ray absorption spectroscopy (HERFD-XAS) $^{24}$ allowed investigation of the structural modification of the intermetallic correlation, as well as the oxidized $\mathrm{Au}$ fraction. The preparation method, coupled with HERFDXAS, allowed us for the first time to understand the chemical effect of the annealing atmosphere on the cluster configurational disorder. Our data suggest a relevant cluster-matrix interaction (i.e., a Au-O coordination) for the smallest clusters, which is likely responsible for electronic surface states and infrared luminescence of Au molecule-like clusters.

\section{Experimental and data analysis}

The matrix used for Au implantations was pure silica (Herasil I by Heraeus), previously doped with Er ions by ion implantation (Er concentration $\sim 0.1$ at $\%$ in a $\sim 70 \mathrm{~nm}$ thick surface layer) and annealed in $\mathrm{N}_{2}$ at $800{ }^{\circ} \mathrm{C}$ for $1 \mathrm{~h}$ to activate Er luminescence. ${ }^{19} \mathrm{Au}$ ions were sequentially implanted at three different energies to obtain a homogeneous concentration depth profile, $\sim 70 \mathrm{~nm}$ thick, superimposed on the Er one. The $\mathrm{Au}$ concentration into the implanted layer spanned more than one order of magnitude, from about 1.5 at\% (a total implantation fluence of $7.0 \times 10^{15}$ ions per $\mathrm{cm}^{2}$ ) to 0.07 at\% (a total implantation fluence of $3.5 \times 10^{14}$ ions per $\mathrm{cm}^{2}$ ). After $\mathrm{Au}$ implantation the samples were annealed for $1 \mathrm{~h}$ at a temperature in the range $300-800{ }^{\circ} \mathrm{C}$ to promote $\mathrm{Au}$ clustering. Both inert $\left(\mathrm{N}_{2}\right)$ and reducing $\left(\mathrm{Ar}+\mathrm{H}_{2}(5 \%)\right)$ atmospheres were used to investigate the chemical effect of the atmosphere on the cluster-matrix interaction and on the cluster growth. The total implantation fluence was measured by Rutherford Backscatter- ing Spectrometry. The samples are labeled to indicate the $\mathrm{Au}$ fluence (with respect to the Er one, i.e. $7 \times 10^{14}$ ions per $\mathrm{cm}^{2}$ ), the annealing atmosphere and temperature: e.g., for the sample Au5N620 the $\mathrm{Au}$ atomic concentration was about 5 times the Er one and the sample was annealed at $T=620{ }^{\circ} \mathrm{C}$ under a nitrogen atmosphere. The labels 'Au5as' and similar ones indicate the as-implanted samples.

The X-ray absorption spectroscopy experiments (both EXAFS-Extended X-ray Absorption Fine Structure and XANESX-ray Absorption Near-Edge Structure) were performed at the Au $\mathrm{L}_{3}$-edge at the ID26 beamline of the European Synchrotron Radiation Facility (Grenoble-France); the monochromator was equipped with a couple of Si (111) crystals. Samples were cooled at $20 \mathrm{~K}$ to avoid X-ray radiation damage and to reduce atomic thermal vibrations. X-ray absorption spectra were recorded in fluorescence mode, using two different experimental setups. In the first one (low resolution) the Au fluorescence signal was recorded by a 13-element high purity Ge detector: the energy resolution, $\approx 200 \mathrm{eV}$, allowed recording of the $\mathrm{Au}$ fluorescence signal superimposed on the low-energy tail of the elastic + Compton scattering. The EXAFS spectra for the series Au5 were all collected in this way. The second setup is very interesting especially for advanced XANES analysis and took advantage of a Ge multi crystal analyzer in Rowland geometry to detect the $\mathrm{Au} \mathrm{L} \alpha_{1}$ fluorescence signal, with an energy resolution of $\approx 2 \mathrm{eV}$, i.e. narrower than the $\mathrm{L} \alpha_{1}$ line of the $\mathrm{Au}$ emission $(5.41 \mathrm{eV}):^{25}$ in this way, the features of the absorption spectrum close to the edge energy are strongly emphasized (High-Energy Resolution-Fluorescence Detected XAS, HERFDXAS). ${ }^{26,27}$ In particular, the absorption signal from the $\mathrm{p} \rightarrow \mathrm{d}$ transition, present in the case of partially oxidized $\mathrm{Au}$ atoms, is well resolved. A second relevant advantage of using a high resolution fluorescence emission setup is that the scattering signal from the matrix is filtered out, improving the overall quality of the collected signal. All the XANES spectra presented and the EXAFS spectra of the series Au5 were collected in this way. One spectrum of the series Au0.5 was also collected in this way for a consistency check. Few spectra of the experimental setup did not allow us to single-out possible edge shifts within $\approx 1 \mathrm{eV}$ : within this limit all the recorded edge positions (maximum of the first derivative) were the same and so all experimental and simulated spectra were accordingly aligned.

The white-line of the $\mathrm{Au} \mathrm{L}_{3}$-edge XANES spectra is related to the electronic $\mathrm{p} \rightarrow \mathrm{d}$ transitions and is the signature of the unoccupied d states, ${ }^{27}$ in our case due to the chemical interaction of $\mathrm{Au}$ atoms with $\mathrm{O}$ atoms of the matrix. Assuming that the white-line area decreases linearly with the increase of the Au metallic fraction $\left(f_{\text {met }}\right)$, we have estimated $f_{\text {met }}$ by fitting the white-line area as a linear combination of the values of the area in the two limit conditions (undetectable $\mathrm{Au}-\mathrm{Au}$ coordination, sample Au0.5as, and undetectable $\mathrm{Au}-\mathrm{O}$ coordination, sample Au0.5N800, see the following). The use of the whiteline area instead of the maximum white-line intensity allows one to correctly consider a possible broadening effect caused by structural disorder. It should be noted that in the presence 
of very small clusters, the white-line in principle could also depend on the hybridization of valence orbitals, which is expected to depend on the cluster size. ${ }^{20}$ Nevertheless in our case, the main changes in the XANES spectra are found to be strongly correlated with the $\mathrm{Au}-\mathrm{O}$ coordination rather than with the cluster size. We will show that samples with a similar cluster size exhibit different white-line intensities.

XANES simulations were performed by the FDMNES code, using the multiple scattering theory based on the muffin-tin approximation on the potential shape. ${ }^{28}$ The muffin-tin radii were tuned to have a $10 \%$ overlap between the different spherical potentials. A relativistic calculation was done and the Hedin-Lundqvist exchange potential was used. As a first step, the XANES spectrum of a Au crystal was calculated, considering in the calculation all the atoms surrounding the absorber within a $8 \AA$ radius sphere. The approximation of non-excited absorbing atoms was used, which better reproduces the experimental data. ${ }^{21}$ A similar calculation with the FEFF8 code $\mathrm{e}^{29,46}$ gave similar results. We have not considered the effect of the structural disorder in the XANES simulations. To simulate the HERFD-XANES spectra, a core-hole broadening of $2 \mathrm{eV}$ was used, i.e. corresponding to the experimental energy resolution, similar to what used by van Bokhoven and co-workers. ${ }^{27}$ Moreover, we have used a maximum value of $5 \mathrm{eV}$ for the final state energy width ${ }^{28}$ that was found to maximize the agreement with the Au foil HERFD-XANES spectrum.

To understand how the characteristic features of the XANES spectrum change with the size of Au clusters, we performed a first set of simulations of very simple structures of $\mathrm{Au}_{n}$ clusters under vacuum, based on the unrelaxed fcc structure: clusters were formed including consecutive complete atomic shells around a central Au atom (so, we considered $n=13,19,43,55$, $79,87,141)$. For each cluster, the simulated absorption spectrum is the average of all the absorption spectra computed for all the atoms in the cluster. The interatomic distance $d_{\mathrm{Au}-\mathrm{Au}}$ for a $n$-atom cluster was scaled according to the size equation: ${ }^{19}$

$$
d_{\mathrm{Au}-\mathrm{Au}}(n)=d_{\mathrm{Au}-\mathrm{Au}}(\infty)\left(1-\frac{A}{n^{\mathrm{B}}}\right)
$$

with $A=0.128 \pm 0.009, B=0.37 \pm 0.03$ and $d_{\mathrm{Au}-\mathrm{Au}}(\infty)=2.88 \AA$, the interatomic distance of the $\mathrm{Au}$ bulk. While structural models of $\mathrm{Au}$ clusters interacting with external molecules for example thiols do exist in the literature ${ }^{30}$ models of Au clusters embedded into solid matrices and in particular in silica are, to our knowledge, not available. So, as a first approximation to include the effect of the matrix on the X-ray absorption spectrum of a Au cluster (with radius $R_{\mathrm{cl}}$ ), the cluster itself was placed in the middle of a spherical hole (with radius $R_{\mathrm{S}}=$ $R_{\mathrm{cl}}+\Delta R$ ) cut out from a $\mathrm{SiO}_{2}$ crystal, as sketched in Fig. 1 . In this way the cluster is surrounded by an empty shell of thickness $\Delta R=R_{\mathrm{s}}-R_{\mathrm{cl}}$ and then by the matrix. An effective medium approximation was used outside a sphere of radius $R_{\text {eff }}>R_{\mathrm{s}}$, to partially compensate for the use of a crystal instead of a disordered $\mathrm{SiO}_{2}$ matrix. Typical used values are $2.75 \AA<R_{\mathrm{S}}$ $<4.00 \AA, \Delta R \simeq 1.7 \AA, R_{\text {eff }} \simeq 7 \AA$. This model is actually very

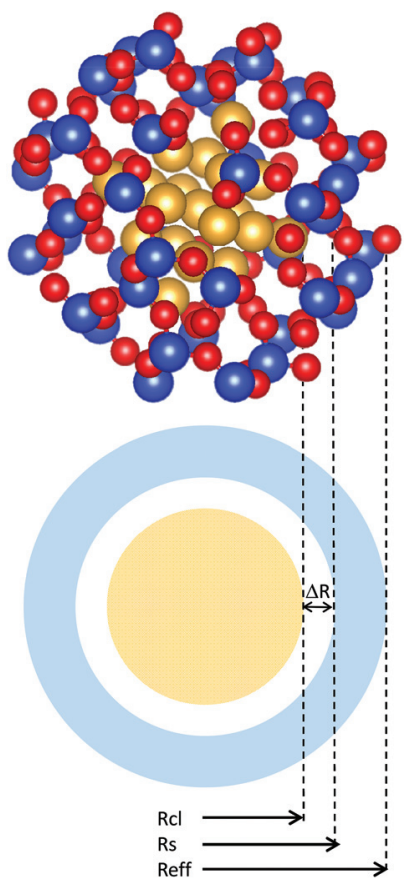

Fig. 1 Sketch of the geometry used for XANES calculations: $R_{\mathrm{cl}}$ is the Au cluster radius, $R_{\mathrm{s}}$ is the radius of the spherical void in the $\mathrm{SiO}_{2}$ matrix and $R_{\text {eff }}$ marks the radius beyond which a medium field potential is used. $\mathrm{Au}$ atoms marked in yellow, $\mathrm{O}$ atoms in red, and $\mathrm{Si}$ atoms in blue.

simple, but it should be considered as a first step to reproduce the main features of the XANES spectra, until the optimized geometries of $\mathrm{Au}$ clusters interacting with silica will become available.

The analysis of the experimental EXAFS spectra was performed using the FEFF8-FEFFIT 2.98 package. $^{29}$ Data were fitted within the single scattering approximation in the $R$-space of the $\mathrm{Au}-\mathrm{O}$ and $\mathrm{Au}-\mathrm{Au}$ correlations. The use of an asymmetric interatomic distance distribution in the present work did not improve significantly the fit quality, and so it was not used. Using the $\mathrm{Au}-\mathrm{Au}$ distance obtained by the fit, the number $n$ of atoms per cluster was estimated from the above reported size equation. ${ }^{19}$ It is worth noting that although this equation derives from Au clusters under vacuum, data available in the literature on supported or embedded Au clusters are in agreement with it. ${ }^{19,31}$ For EXAFS data with an intrinsically limited extension in the $k$-range $(k=$ photoelectron wavenumber) it is well known that the overall amplitude of the $\mathrm{Au}-\mathrm{Au}$ coordination signal (accounting for the coordination number and metallic fraction $f_{\text {met }}$ ) and the Debye-Waller factor are correlated parameters and often do not allow one to properly estimate disorder and the oxidized fraction at the same time. ${ }^{19,31}$ In the present case, we have estimated the coordination number according to the cluster size and the oxidized fraction using the area of the white-line, as explained above. Only the highlighted gray part of the white-line shown in Fig. 2(a) was considered, since the right-hand side was found to be slightly influenced by the cluster size. In this way 


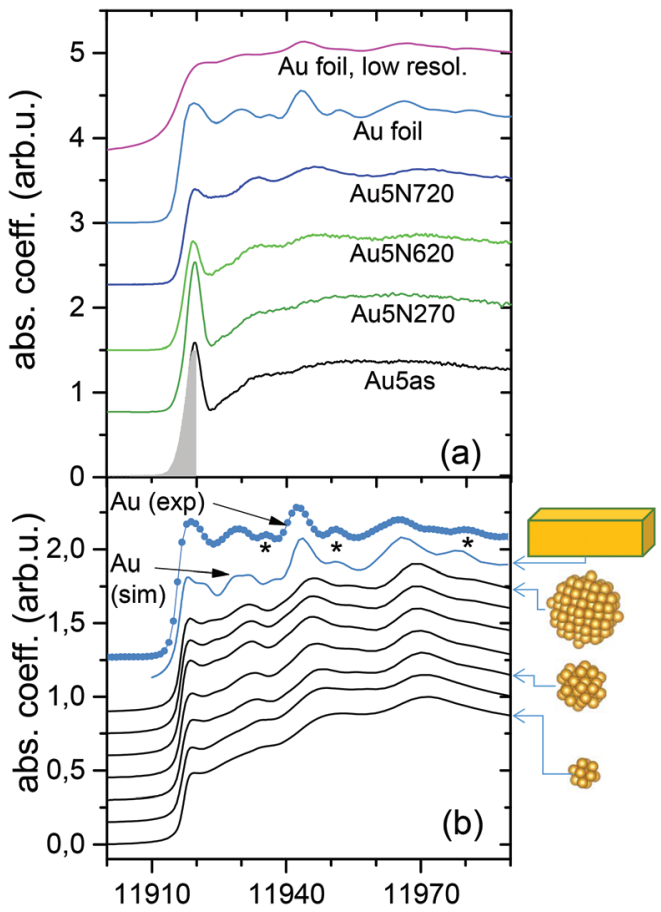

Fig. 2 (a) HERFD-XANES normalized spectra of Au implanted Er-doped silica (Au5 series), after annealing in $\mathrm{N}_{2}$ at different temperatures; Au foil spectra, measured with low and high resolution, are reported for comparison. The region of the white-line used for evaluating the oxidized fraction is marked in gray. (b) XANES simulation of $\mathrm{Au}_{n}$ clusters under vacuum: from bottom to top $n=13,19,43,55,79,87$, and 141 (black lines). The simulation of $\mathrm{Au}$ foil is also reported (color line) and compared with the corresponding experimental spectrum (markers). Asterisks indicate the features that can be simulated only by considering clusters of at least $141 \mathrm{Au}$ atoms.

the Debye-Waller factor for the $\mathrm{Au}-\mathrm{Au}$ coordination was estimated by the fitting procedure. Then, the effective coordination number $N_{\text {eff }}$ of the Au atomic shell has been fixed to the value $N_{\text {eff }}=N_{\mathrm{cl}} \cdot f_{\text {met }}$, where $N_{\mathrm{cl}}$ is the coordination number pertaining to a cluster whose size is determined by the $\mathrm{Au}-\mathrm{Au}$ distance through the size equation. ${ }^{19,32}$ This procedure is new and takes advantage of the HERFD setup with respect to the study performed in our previous paper ${ }^{19}$ and allowed us to discuss more reliably the cluster structural disorder.

\section{Results and discussion}

We have recently shown that small Au clusters in silica exhibit photoluminescence bands one of which in the spectral region around $980 \mathrm{~nm}$ is thought to originate from electronic surface states of Au clusters. ${ }^{11}$ So, to shed light on the interaction of $\mathrm{Au}$ clusters with the surrounding silica matrix, we discuss here the results of the HERFD-XAS experiment.

In Fig. 2(a) the $\mathrm{Au} \mathrm{L}_{3}$-edge HERFD-XANES spectra of $\mathrm{Au}$ foil and of a series of $\mathrm{Er}+\mathrm{Au}$ co-implanted silica samples are shown, as the representative of all the measured spectra. As discussed in the previous section, the white-line in the HERFD-XANES spectrum is directly related to the number of unoccupied d-states. In all sample series its intensity is maximum for the as-implanted sample and progressively decreases upon annealing at increasing temperatures. Together with the decrease of the white-line, the features typical of the Au metallic phase become more evident. The spectrum of $\mathrm{Au}$ foil recorded in fluorescence mode with a standard (low) resolution is also reported: the advantage of using the high-resolution mode to gain information on the non-metallic $\mathrm{Au}$ fraction is evident. With respect to the HERFD-XANES spectrum of bulk $\mathrm{Au}$, the features above the absorption edge are less visible for the considered samples, due to the small size of the embedded Au aggregates. They are located at higher energy, due to a size-related interatomic distance contraction typical of nanostructures. Within the experimental accuracy, we do not observe any significant edge shift.

The following HERFD-XANES analysis focuses first on $\mathrm{Au}$ clusters under vacuum and then on Au clusters in $\mathrm{SiO}_{2}$.

In Fig. 2(b) the simulated spectra for $\mathrm{Au}_{n}$ clusters under vacuum $(n=13, \ldots, 141)$ are reported. As shown for the experimental spectra the main features of the X-ray absorption spectra become more evident and their position shifts towards lower energy as the cluster size increases. The HERFD-XANES spectrum of Au foil is compared with the corresponding simulation that reproduces nicely the main features of the experimental one (Fig. 2(b)). The structures in the Au foil spectrum indicated with an asterisk in the figure mark the onset of long range order and in our simulations become visible only for clusters larger than 141 atoms (i.e., $1.5 \mathrm{~nm}$ sized clusters).

The effect of the surrounding matrix has been included in the simulations using the results of the EXAFS analysis. While the full EXAFS analysis is reported below, here we anticipate a few points that are relevant for the present discussion.

For the sample with the lowest $\mathrm{Au}$ concentration and annealed at $800{ }^{\circ} \mathrm{C}$ (sample Au0.5N800), the EXAFS analysis shows only the $\mathrm{Au}-\mathrm{Au}$ coordination: the measured $\mathrm{Au}-\mathrm{Au}$ distance is related to the formation of 13-atom clusters (about $0.7 \mathrm{~nm}$ size). The coordination number for the Au shell is $4.9 \pm$ 0.5 , in perfect agreement with the one predicted for 13 atom clusters $\left(N_{\mathrm{Au}}=5\right)$. The absence of a $\mathrm{Au}-\mathrm{O}$ coordination indicates a faint interaction of Au clusters with the matrix. In Fig. 3(a) the HERFD-XANES spectrum of the sample Au0.5N800 is compared with two simulations for a $\mathrm{Au}_{13}$ cluster

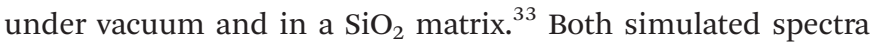
have features similar to the experimental one. Nevertheless, the presence of the matrix allows one to better reproduce the general shape of the X-ray absorption spectrum: in particular, in the region 11920-11950 eV the simulated spectrum is much more similar to the experimental one if the matrix is included in the structural model.

In Fig. 3(b) the HERFD-XANES spectrum of the sample Au5N600 (0.75 nm size cluster and Au-O coordination present, according to EXAFS) is shown and compared with a simulation based on the EXAFS results of $\mathrm{Au}_{19}$ cluster in silica: the simulation reproduces the main structures of the spectrum. 


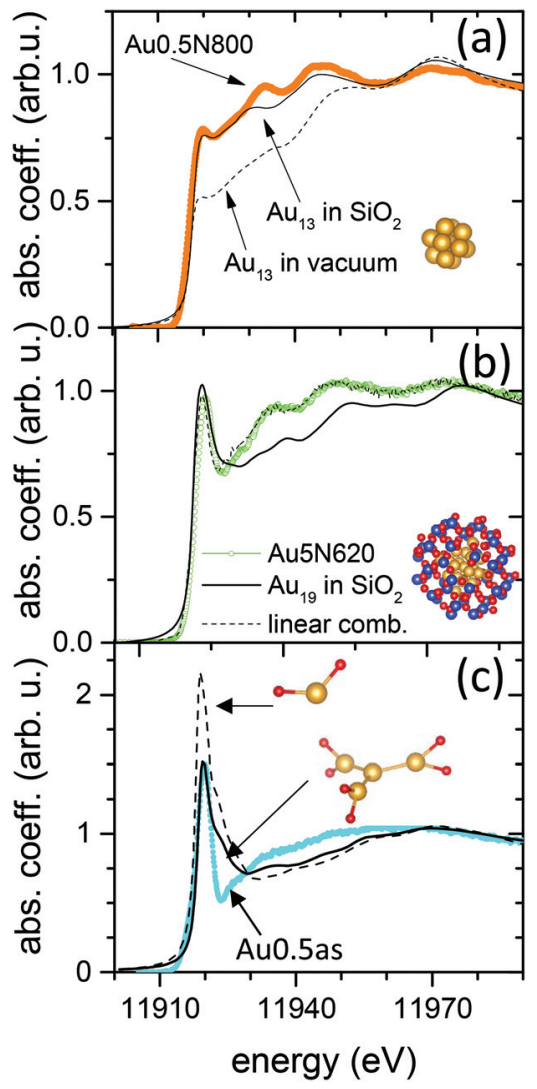

Fig. 3 (a) HERFD-XANES experimental spectrum (markers) of low-dose $\mathrm{Au}$ implanted Er-doped silica after $800{ }^{\circ} \mathrm{C}$ annealing (sample Au0.5N800), compared with the simulated spectra of a 13-atom cluster in $\mathrm{SiO}_{2}$ and under vacuum. (b) HERFD-XANES experimental spectrum (markers) of the sample Au5N600, compared with the simulated spectrum of a 19-atom cluster in $\mathrm{SiO}_{2}$, and with a linear combination of experimental spectra in (a) and (c). (c) HERFD-XANES experimental spectrum of low-fluence Au implanted Er-doped silica (sample Au0.5as), compared with two spectra calculated from the sketched structures (red: O atoms, blue: Si atoms, yellow: Au atoms).

Considering now the as-implanted sample of the series with the lowest Au concentration (Au0.5as), the EXAFS analysis shows that the $\mathrm{Au}-\mathrm{Au}$ coordination is below the detectable limit and the only present signal is from the $\mathrm{Au}-\mathrm{O}$ correlation, indicating that all $\mathrm{Au}$ atoms in the implanted layer are oxidized. The coordination number $N_{\mathrm{O}}$ for the oxygen shell is about 2 and the average $\mathrm{Au}-\mathrm{O}$ distance is $2 \AA$. Correspondingly, the white-line of the HERFD-XANES spectrum is the highest. In Fig. 3(c) the HERFD-XANES spectrum of the sample Au0.5as is compared with two simulations for (i) one Au atom surrounded by two $\mathrm{O}$ atoms and for (ii) one extended cluster combining $\mathrm{Au}-\mathrm{O}$ configurations as in (i) with some $\mathrm{Au}-\mathrm{Au}$ coordination. The two selected structures can be considered as representative of two distinct configurations pertaining to the early steps of $\mathrm{Au}$ nucleation and growth in silica: with respect to configuration (i), configuration (ii) represents the onset of the Au-Au metal-metal coordination which will be dominating upon annealing at high temperatures. In both cases a $\mathrm{SiO}_{2}$ matrix surrounds the cluster and the Au-matrix distance is longer than $3 \AA$. Even if the structures (i) and (ii) are too simple to be expected to correctly reproduce the details of experimental data, a few points can still be suggested by this basic approach. For the structure (i) the agreement with the experimental spectrum is modest and does not improve if the $\mathrm{O}-\widehat{\mathrm{Au}}-\mathrm{O}$ angle is changed (tested in the $60-128^{\circ}$ range). The white-line of the simulation is too intense compared to the experimental data, suggesting a higher average electronic doccupancy of Au atoms in the sample compared to the simulated structure. A better (although not full) agreement is obtained if an extended structure of interconnected $\mathrm{Au}$ and $\mathrm{O}$ atoms is considered with embryos of $\mathrm{Au}-\mathrm{Au}$ coordination (ii), for example the one in Fig. 3(c). This and the simulations performed on other similar configurations may suggest that even when the majority of $\mathrm{Au}$ atoms are oxidized (only the $\mathrm{Au}-\mathrm{O}$ coordination is detected by EXAFS in the Au0.5as sample), the $\mathrm{Au}$ atoms participate into partially metallic aggregates of 2-4 $\mathrm{Au}$ atoms, strongly connected with the matrix via $\mathrm{Au}-\mathrm{O}$ coordinations. A variety of these structures is likely to occur, resulting in strong configurational disorder and in an experimentally undetectable $\mathrm{Au}-\mathrm{Au}$ coordination, spread over a wide range of $\mathrm{Au}-\mathrm{Au}$ distances and characterized by a low coordination number. Therefore, these simulations seem to indicate that the main structural units are composed of Au atoms coordinated to $\mathrm{O}$ atoms, in the presence of some $\mathrm{Au}-\mathrm{Au}$ coordination. This picture produces better results with respect to the one in which the single structural units (oxidized $\mathrm{Au}$ atoms) are completely separated from each other (Fig. 2(b)). In this framework, clusters in the Au5N600 sample (Fig. 3(b)) can be sketched as formed of a core of metal atoms surrounded by a shell of $\mathrm{Au}$ atoms covalently connected with the matrix through $\mathrm{O}$ atoms. Correspondingly, the HERFD-XANES spectrum of Au5N600 is very similar to a linear combination of the spectra of Au05N800 (weight 0.73) and Au05as (weight 0.27) samples.

Overall, the HERFD-XANES analysis suggests that a $\mathrm{Au}$ cluster can be sketched as an effective core-shell system in which the Au atoms in the 'core' develop a metallic character (i.e., Au metallic fraction in our measurements), whereas the $\mathrm{Au}$ atoms in the 'shell' can retain a partially covalent bond with $\mathrm{O}$ atoms of the matrix. As the cluster grows the 'core' metallic Au atoms dominate over the 'shell' ones in the XAS signal. This core-shell-like picture is consistent with the model of thiol-capped $\mathrm{Au}_{n}$ clusters. ${ }^{30}$

The following full XAS analysis allows one to study how Au cluster nucleation and the first steps of cluster growth are influenced by the surrounding silica matrix.

Some representative HERFD-EXAFS spectra are reported in Fig. 4(a), where they are compared with the spectrum of Au foil measured in transmission mode at the same temperature. The analysis showed only the presence of a $\mathrm{Au}-\mathrm{Au}$ and $\mathrm{Au}-\mathrm{O}$ coordination, while no $\mathrm{Au}-\mathrm{Er}$ or $\mathrm{Au}-\mathrm{Si}$ coordinations are detected. Fits are reported in Fig. 4(b-d). In particular, from Fig. 4(b and c), the two $\mathrm{Au}-\mathrm{O}$ and $\mathrm{Au}-\mathrm{Au}$ coordinations are visible, while no significant signal from coordination shells 

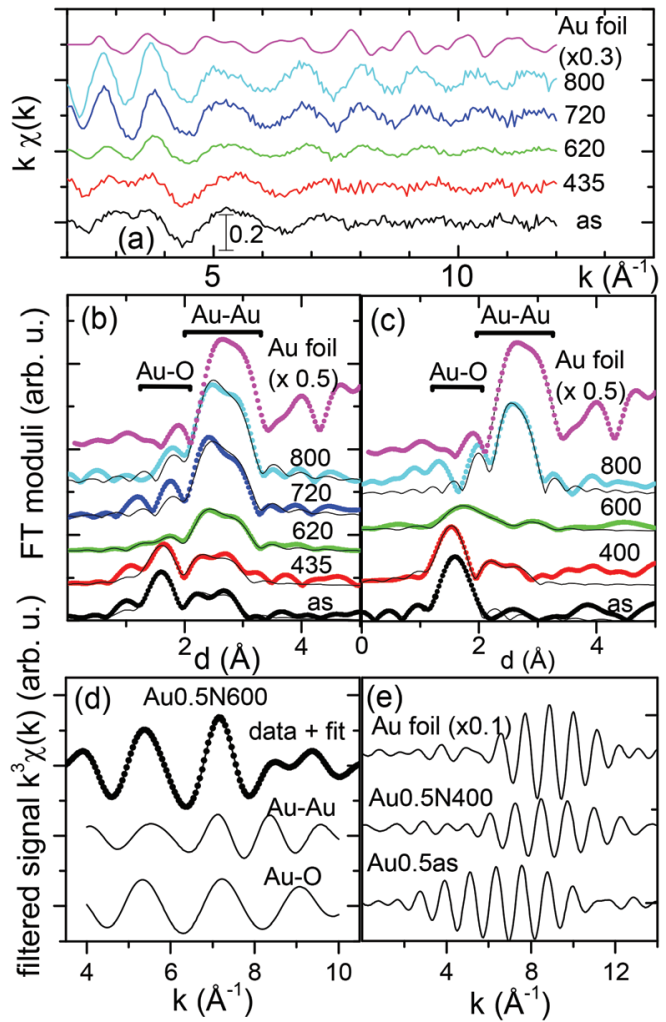

Fig. 4 (a) HERFD-EXAFS spectra (series Au5) and (b, c) Fourier transform moduli and relative fits of the $\mathrm{Au}$ implanted Er-doped silica samples annealed under a neutral atmosphere: series Au5 (b), and series Au0.5 (c). The corresponding signals for $\mathrm{Au}$ foil are also reported. Numbers mark the annealing temperature in ${ }^{\circ} \mathrm{C}$. (d) Fourier filtered signal for the Au0.5N600 sample, superimposed on the corresponding best fit curve. $\mathrm{Au}-\mathrm{O}$ and $\mathrm{Au}-\mathrm{Au}$ correlations are shown. (e) EXAFS signals after processing by Fourier filtering in the range $R=2.2-3.2 \AA$ of the samples Au0.5as and Au0.5N400, compared to the corresponding spectrum from $\mathrm{Au}$ foil: the $\mathrm{Au}-\mathrm{Au}$ coordination signal, visible upon $400{ }^{\circ} \mathrm{C}$ annealing, is not present in the as-implanted sample.

beyond the first one is present. For all the investigated sample series, the Au-O coordination upon annealing at $T>600{ }^{\circ} \mathrm{C}$ is too small to be quantitatively analyzed. The $\mathrm{Au}-\mathrm{Au}$ distance is in all cases significantly shorter than in the bulk, indicating a small size of the aggregates. In Fig. 4(e) the EXAFS signals, filtered in the range pertaining to the intermetallic coordination, are shown for the samples Au0.5as and Au0.5N400. The comparison with the same signal for Au foil clearly shows that the $\mathrm{Au}-\mathrm{Au}$ correlation is undetectable in the as-implanted sample and is present upon $400{ }^{\circ} \mathrm{C}$ annealing. The results of the XAS analysis are reported in Table 1 (Au-O coordination) and in Fig. 5(a-c) (Au-Au coordination).

Data in Table 1 indicate a relevant interaction of Au clusters with $\mathrm{O}$ atoms in the matrix. In principle the $\mathrm{Au}-\mathrm{O}$ coordination could be pertaining to a fraction of Au ions dispersed into the matrix and oxidized. Nevertheless, if this would be the case, the effective coordination number of this $\mathrm{O}$ shell would be about 3-4 for at least two cases, to be compared with the coordination number of $\approx 2$ experimentally found for other
Table 1 Results of the EXAFS analysis for the Au-O coordination; $N_{O}$ is the coordination number, $d_{\mathrm{Au}-\mathrm{O}}$ is the interatomic distance; the DebyeWaller factor of the Au-O coordination is $\sigma^{2}=(5 \pm 1) \times 10^{-3} \AA^{2}$. Data on $\mathrm{Au}-\mathrm{Au}$ coordination are collected in Fig. 5

\begin{tabular}{llll}
\hline Sample & & $N_{\mathrm{O}}$ & $d_{\mathrm{Au}-\mathrm{O}}(\AA)$ \\
\hline $\mathrm{Au0.5}$ & $\mathrm{as}$ & $1.9 \pm 0.4$ & $2.00 \pm 0.02$ \\
$\mathrm{Au} 0.5$ & $\mathrm{~N} 400$ & $1.6 \pm 0.4$ & $1.93 \pm 0.02$ \\
$\mathrm{Au} 0.5$ & $\mathrm{~N} 600$ & $0.7 \pm 0.3$ & $2.07 \pm 0.02$ \\
$\mathrm{Au} 0.5$ & $\mathrm{~N} 800$ & - & - \\
$\mathrm{Au} 0.5$ & $\mathrm{H} 100$ & $1.9 \pm 0.4$ & $1.94 \pm 0.02$ \\
$\mathrm{Au} 0.5$ & $\mathrm{H} 400$ & $0.8 \pm 0.3$ & $1.99 \pm 0.03$ \\
$\mathrm{Au} 0.5$ & $\mathrm{H} 600$ & $0.7 \pm 0.2$ & $2.09 \pm 0.03$ \\
$\mathrm{Au} 0.5$ & $\mathrm{H} 800$ & - & - \\
$\mathrm{Au} 5$ & $\mathrm{as}$ & $1.6 \pm 0.4$ & $2.00 \pm 0.02$ \\
$\mathrm{Au} 5$ & $\mathrm{~N} 435$ & $1.3 \pm 0.3$ & $1.99 \pm 0.02$ \\
$\mathrm{Au} 5$ & $\mathrm{~N} 620$ & $0.4 \pm 0.2$ & - \\
$\mathrm{Au} 5$ & $\mathrm{~N} 720$ & - & - \\
$\mathrm{Au} 5$ & $\mathrm{~N} 800$ & - & \\
$\mathrm{Au}_{2} \mathrm{O}_{3}$ & 4 & $1.93-2.07$ &
\end{tabular}

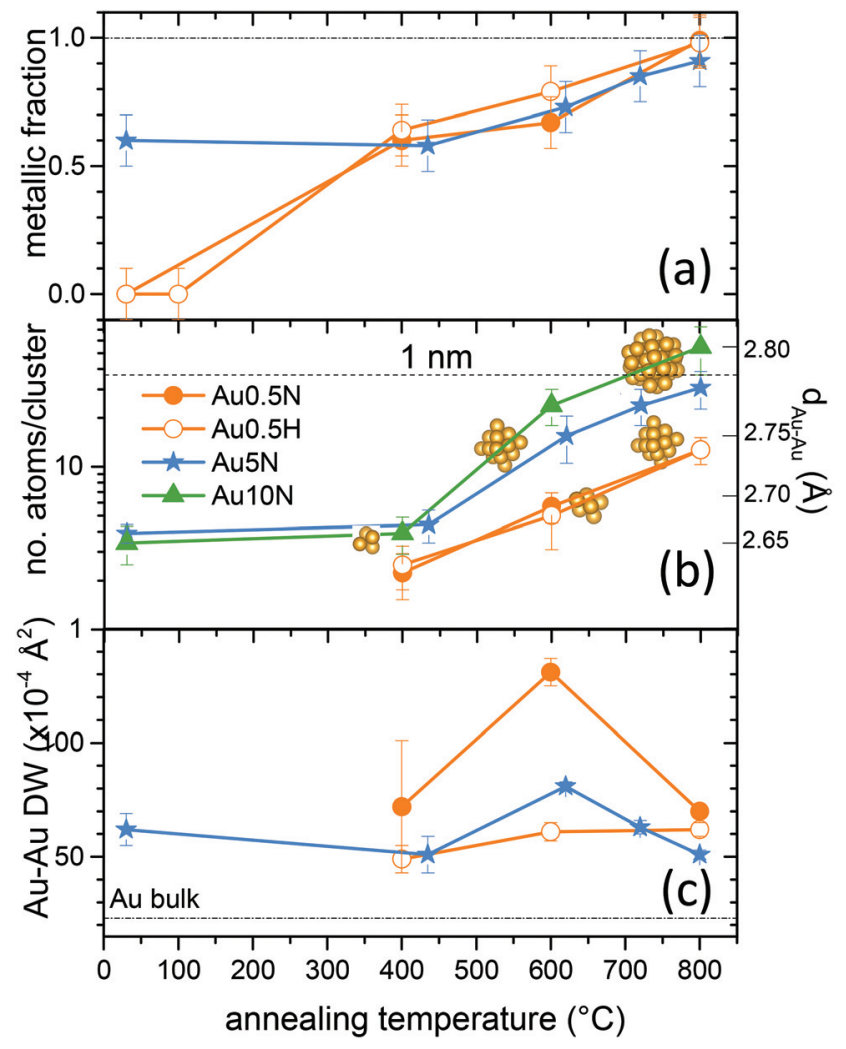

Fig. 5 HERFD-XAS results for Au clusters as a function of annealing temperature. (a) Metallic fraction, (b) average number of atoms per cluster (left vertical axis) and $\mathrm{Au}-\mathrm{Au}$ interatomic distance (right hand vertical axis), (c) Debye-Waller factor of the Au-Au correlation in the different $\mathrm{Er}+\mathrm{Au}$-implanted silica slides.

noble metal ions, such as $\mathrm{Ag}$, introduced in solid glass matrices. ${ }^{17}$ Considering also that the Gibbs free energy for $\mathrm{Au}-$ oxide formation is the highest among those of transition metal oxides, a O coordination at the cluster surface seems to be 
more reasonable, further supporting the picture of core-shelllike clusters. The Au-O coordination distance varies in a nonmonotonic way (Table 1) with the annealing temperature. It has to be observed that, for very small clusters as those considered here, practically all atoms are at the cluster surface, so that in the effective core-shell picture of the cluster discussed above, the 'shell' part is dominating. To this respect, density functional theory (DFT) calculations for Au atoms and dimers trapped on various defect sites of amorphous silica found that the $\mathrm{Au}-\mathrm{O}$ equilibrium distance depends on the defect type, varying a few \% around a value of about $2.05 \AA{ }^{16}$ In particular, DFT calculations estimate a Au-O distance of 2.02-2.04 $\AA$ for $\mathrm{Au}$ and $\mathrm{Au}_{2}$ onto non-bridging oxygen defects of a flat silica surface. ${ }^{16}$ Similar DFT calculations for one $\mathrm{O}$ atom on specific $\mathrm{Au}$ crystallographic surfaces showed a Au-O equilibrium distance that depends on the local structure of the Au surface and is in the same range. ${ }^{34-36}$ The variety and the range of $\mathrm{Au}-\mathrm{O}$ distances found in our case suggest a similar scenario, where $\mathrm{Au}$ atoms at the cluster surface contribute to the $\mathrm{Au}-\mathrm{O}$ correlation. This system has some analogy to $\mathrm{Au}$-thiolate clusters, for which EXAFS analyses at the Au-edge evidenced a $\mathrm{Au}-\mathrm{Au}$ correlation ( $\mathrm{Au}$ atoms in the core) and a $\mathrm{Au}-\mathrm{S}$ correlation (Au atoms in the shell). ${ }^{18,37,38}$ In general the coordination number for the $S$ shell varies from less than 1 to 2 and the $\mathrm{Au}-\mathrm{S}$ coordination can involve up to two external shells of $\mathrm{Au}$ atoms in the cluster. ${ }^{37,38}$ In our case the average results support a similar picture, with Au atoms at the surface coordinating a few $(\leq 2) \mathrm{O}$ atoms. Some experiments on $\mathrm{Au}$-thiolate clusters evidenced the formation of icosahedral structures. ${ }^{18,37,38}$ Their possible formation in our case, if any, is likely hindered by the co-presence of a variety of other different structures.

The XAS structural results allow estimation of the energy barrier for the Au metal nucleation in Er-implanted silica. Considering the Au0.5 sample series, i.e. the one with the lowest investigated Au concentration, as previously noted, the nucleation of $\mathrm{Au}$ into metal clusters, undetected in the as-implanted sample, first occurred upon inert annealing at $400{ }^{\circ} \mathrm{C}$ (sample Au0.5N400, Fig. 4(e)), with a measured Au-Au distance of 2.60 $\pm 0.02 \AA$, to be compared with the average one calculated for Au dimers and trimers of $2.54 \AA$ and $2.57 \AA$, respectively ${ }^{19}$ and with the bulk value of $2.88 \AA$. The measured $\mathrm{Au}-\mathrm{Au}$ distance corresponds to the formation of a cluster of 2-4 atoms, i.e., the cluster whose size is the critical one. ${ }^{19}$ Considering that the nucleation energy barrier has been overcome by $400{ }^{\circ} \mathrm{C}$ annealing, we can approximately estimate that the maximum Gibbs free energy for the heterogeneous nucleation of $\mathrm{Au}$ in Er-doped silica is $k_{\mathrm{B}} T \approx 0.06 \mathrm{eV}$ per atom. As previously noted, this should be considered as the energy barrier to move from an oxidized phase, in which $\mathrm{Au}$ atoms with embryonic $\mathrm{Au}-\mathrm{Au}$ correlation are strongly connected to the matrix, towards clusters characterized by a well-defined $\mathrm{Au}-\mathrm{Au}$ coordination distance. The energy barrier for this heterogeneous nucleation is, as expected, much lower than the corresponding one for the homogeneous nucleation $\left(\approx 2 \mathrm{eV}\right.$ per atom $\left.{ }^{39}\right)$ : this occurs because Au nucleation in the implanted silica is a pheno- menon assisted by defects (e.g., non-bridging oxygens, $\mathrm{E}^{\prime}$ centers) that act as nucleation centers for the metallic phase. ${ }^{40}$ For this reason, the nucleation energy barrier is also expected to depend on the Au fluence (i.e., on the defect density) and maybe slightly on the presence of Er ions embedded in the same matrix. Nevertheless, some measurements performed on Au clusters prepared under similar conditions into pure silica suggest that the nucleation energy barrier is of the same order. Moreover, as previously noted, the HERFD-XAS analysis did not detect any Au-Si coordination, suggesting a preferential interaction of $\mathrm{Au}$ with non-bridging oxygens rather than $\mathrm{E}^{\prime}$ centers.

After nucleation, clusters grow up to $1.1 \mathrm{~nm}$ (55 atoms, Au10N800, the highest investigated $\mathrm{Au}$ concentration), as shown in Fig. 5(b). Their average size is easily tuned by varying the implantation fluence and the annealing temperature. The marked growth observed at higher Au concentration depends on both the relatively high Au concentration and on the higher damage level of the matrix, related to the 'high' implantation fluence: indeed, it is known that dopant diffusion during implantation is enhanced by irradiation. ${ }^{40}$ As expected, the Debye-Waller (DW) factor of the Au-Au coordination for all the samples is significantly larger than the value for the bulk phase $\left(\sigma^{2}=23 \times 10^{-4} \AA^{2}\right)$ or for supported $2 \mathrm{~nm}$ clusters $\left(\sigma^{2}=\right.$ $\left.40 \times 10^{-4} \AA^{2}\right)^{41}$ measured at the same temperature. Regarding the chemical effect of the annealing atmosphere, it is interesting to note that contrary to what happens at higher $\mathrm{Au}$ fluences $(\approx 1.5$ at\% $),{ }^{19}$ in the case of the lowest investigated $\mathrm{Au}$ concentration, the cluster size and the metallic fraction are not considerably affected by the annealing atmosphere (see Fig. 5 (a and b)). Indeed, while at high Au fluence the Au aggregation depends both on the Au diffusion and on the chemical interaction of the dispersed metal with the oxygen atoms in the matrix, ${ }^{19}$ at low Au fluence (samples Au0.5) the dominant parameter driving clustering is the very low $\mathrm{Au}$ concentration $(\approx 0.07$ at $\%)$ that limits the cluster growth. Nevertheless, the annealing atmosphere can influence the cluster structure through a chemical interaction of the $\mathrm{Au}$ atoms at the cluster surface with $\mathrm{O}$ atoms from the matrix. This is more evident on the samples doped with the lowest Au concentration $(\approx 0.07 \%$, Au0.5 series), for which essentially all Au atoms forming clusters are at the cluster surface. So, the following discussion focuses on these samples.

We observe that after $600{ }^{\circ} \mathrm{C}$ annealing the $\mathrm{Au}-\mathrm{Au} \mathrm{DW}$ factor is much higher for annealing under a neutral atmosphere than under a reducing one (see Fig. 5(c)), despite the average cluster size and the metallic fraction being the same (Fig. 5(a and b)). Since all the spectra are measured at the same temperature $(20 \mathrm{~K})$, a higher DW factor indicates higher structural disorder. In this case the high DW observed for the Au0.5N600 sample is most likely related to high configurational disorder of Au clusters. ${ }^{42}$ It is worth observing that also at higher Au concentration (series Au5) $600{ }^{\circ} \mathrm{C}$-neutral annealing seems to maximize the structural disorder (Fig. 5(c)). In this range of temperatures the silica matrix starts to recover from radiation damage ${ }^{43}$ with strong structural rearrange- 
ments that can induce the temporary formation of a variety of cluster configurations. In particular, upon annealing in $\mathrm{H}_{2}$ : $\mathrm{Ar}$, $\mathrm{H}$ atoms from the atmosphere favor damage recovery of the matrix through the passivation of Si dangling bonds. ${ }^{40,44}$ In this way the most stable Au cluster configuration is favored, resulting in relatively low structural disorder of $\mathrm{Au}$ sites. In contrast, at $600{ }^{\circ} \mathrm{C}$ nitrogen permeation and diffusion in the matrix is expected to be poor, as demonstrated for oxygen. ${ }^{40} \mathrm{It}$ is reasonable to think that higher configurational disorder of the matrix imposes structural constraints to the Au clusters, leading to the formation of a variety of metastable cluster structural configurations. At higher annealing temperature clusters grow and only the most stable configurations survive.

Overall the results indicate that despite the 'noble' character of $\mathrm{Au}$ atoms, their interaction with the implanted silica matrix is evident. In the as-implanted case, the $\mathrm{Au}-\mathrm{O}$ interaction, if coupled to a low Au concentration, prevents the formation of clusters with fully metallic character. As the cluster starts to grow, a well defined and size-dependent $\mathrm{Au}-\mathrm{Au}$ interatomic distance is detected; nevertheless during the first steps of growth (inert annealing), the cluster-matrix interaction can induce strong structural disorder of the metal-metal coordination.

Fig. 6 summarizes the proposed nucleation mechanism for Au clusters in implanted silica. The interaction of the Au clusters with the matrix is clearly favored for small cluster sizes because of the high fraction of Au atoms at the cluster surface. Anyway, Au atoms and dimers onto a silica surface are known to anchor at silica defects. ${ }^{16}$ As noted above, the experimental data indicate a cluster-matrix interaction mediated by $\mathrm{O}$ atoms, most likely non-bridging oxygens. Thus it seems

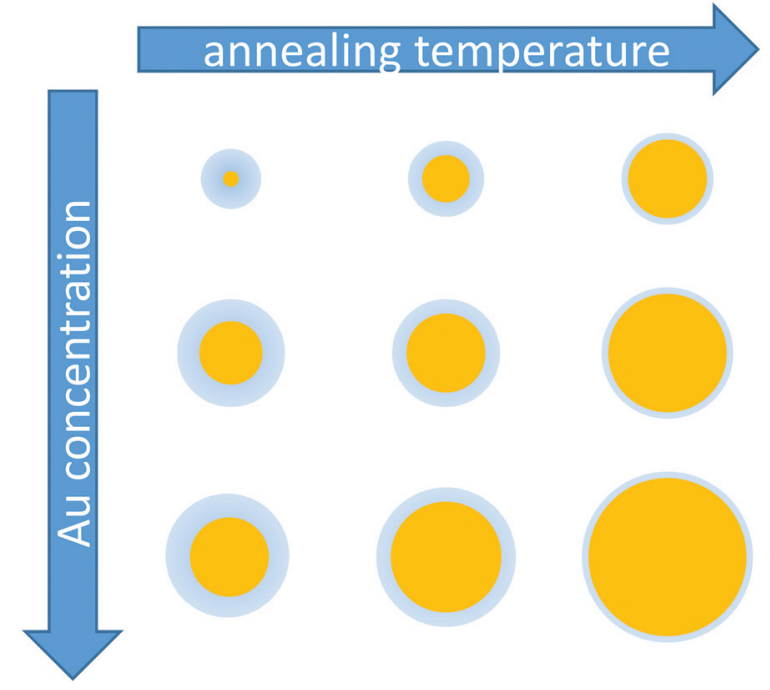

Fig. 6 Sketch of the proposed nucleation process of Au clusters (yellow circle) in silica: Au-O coordination (blue shell) is favored for a small cluster size and low annealing temperature. A thicker shell indicates a higher $\mathrm{Au}-\mathrm{O}$ coordination at the cluster surface. reasonable that, for a definite cluster size, a stronger clustermatrix interaction occurs when silica defects are not completely recovered by annealing. Indeed, this is what we observe for the samples Au5N600 and Au0.5N800: both exhibit Au clusters of about the same size ( $\approx 15$ atoms), but only in the sample annealed at lower temperature (Au5N620) the Au-O coordination is detected by EXAFS. So, a highly defective silica matrix seems to favor the Au-O covalent interaction. During annealing at $600{ }^{\circ} \mathrm{C}$ and higher temperatures the silica starts to recover from defects and the $\mathrm{Au}-\mathrm{O}$ coordination progressively vanishes. This picture is consistent with the photoluminescence results on the same samples ${ }^{11}$ or on ultrasmall capped $\mathrm{Au}$ clusters, ${ }^{45}$ as well as with the general structure of thiol-capped $\mathrm{Au}_{n}$ clusters. ${ }^{30}$

\section{Conclusions}

The very early steps of Au metal cluster formation in Er-doped silica have been investigated by high resolution fluorescence detected X-ray absorption spectroscopy. A combined EXAFS and HERFD-XANES analysis shows that Au cluster nucleation occurs upon ion implantation starting from a few $\mathrm{Au}$ and $\mathrm{O}$ atoms covalently interconnected, in the presence of embryonic $\mathrm{Au}-\mathrm{Au}$ correlation for the lowest investigated $\mathrm{Au}$ concentrations. The first Au clusters characterized by a well defined $\mathrm{Au}-\mathrm{Au}$ coordination distance form upon $400{ }^{\circ} \mathrm{C}$ inert annealing, allowing us to estimate that the Gibbs free energy for this heterogeneous nucleation is not higher than $0.06 \mathrm{eV}$ per atom. This value, much lower than for the homogeneous Au nucleation, suggests that the cluster nucleation is assisted by matrix defects, most likely non-bridging oxygen atoms. The formed subnanometer $\mathrm{Au}$ clusters can be sketched as an effective core-shell system in which the Au atoms of the 'core' develop a metallic character, whereas the Au atoms in the 'shell' can retain a partially covalent bond with $\mathrm{O}$ atoms of the silica matrix. High structural disorder at the $\mathrm{Au}$ site is found upon neutral annealing at a moderate temperature $\left(600{ }^{\circ} \mathrm{C}\right)$, likely driven by the configurational disorder of the defective silica matrix that favors the formation of a variety of cluster structural configurations. A suitable choice of the Au concentration and annealing temperature allows tailoring of the Au cluster size in the sub-nanometer range (i.e., from 3-4 atoms per cluster to about $1 \mathrm{~nm}$ cluster). The interaction of the $\mathrm{Au}$ cluster surface with the surrounding silica matrix is likely responsible for the infrared luminescence previously reported on the same systems.

\section{Acknowledgements}

We acknowledge the European Synchrotron Radiation Facility for provision of the beamtime and the assistance of Jannine Grattage and Marcin Sikora during measurements at the beamline ID26. 


\section{References}

1 D. Widmann and R. J. Behm, Acc. Chem. Res., 2014, 47, 740-749.

2 S. Lee, C. Fan, T. Wu and S. L. Anderson, J. Chem. Phys., 2005, 123, 124710.

3 M.-C. Daniel and D. Astruc, Chem. Rev., 2004, 104, 293-346.

4 G. Mattei, P. Mazzoldi, M. Post, D. Buso, M. Guglielmi and A. Martucci, Adv. Mater., 2007, 19, 561-564.

5 J. Anker, W. Paige Hall, O. Lyandres, N. Shah, J. Zhao and R. Van Duyne, Nat. Mater., 2008, 7, 442-453.

6 X. Wu, T. Ming, X. Wang, J. Wang and J. Chen, ACS Nano, 2010, 4, 113-120.

7 H. Atwater and A. Polman, Nat. Mater., 2010, 9, 205-213.

8 R. Bardan, L. Hedges, C. Pint, S. Whitelam and J. Urban, Nat. Mater., 2013, 12, 905-912.

9 U. Heiz and E. Bullock, Mater. Chem., 2003, 14, 564577.

10 J. Zheng, P. Nicovich and R. Dickinson, Annu. Rev. Phys. Chem., 2007, 58, 409-431.

11 T. Cesca, B. Kalinic, C. Maurizio, C. Scian, G. Battaglin, P. Mazzoldi and G. Mattei, Nanoscale, 2013, 6, 17161724.

12 J. Polte, X. Tuaev, M. Wuithschick, A. Fischer, A. F. Thuenemann, K. Rademann, R. Kraehnert and F. Emmerling, ACS Nano, 2012, 6, 5791-5802.

13 A. Simo, J. Polte, N. Pfänder, U. Vainio, F. Emmerling and K. Rademann, J. Am. Chem. Soc., 2012, 134, 1882418833.

14 M. Harada and H. Einaga, Langmuir, 2007, 23, 65366543.

15 M. Eichelbaum, K. Rademann, A. Hoell, D. M. Tatchev, W. Weigel, R. Stösser and G. Pacchioni, Nanotechnology, 2008, 19, 135701.

16 A. Del Vitto, G. Pacchioni, K. H. Lim, N. Rösch, J.-M. Antonietti, M. Michalski, U. Heiz and H. Jones, J. Phys. Chem. B, 2005, 109, 19876-19884.

17 C. Maurizio, A. Quaranta, E. Ghibaudo, F. D'Acapito and J.-E. Broquin, J. Phys. Chem. C, 2009, 113, 8930-8937.

18 R. E. Benfield, D. Grandjean, M. Kröll, R. Pugin, T. Sawitowski and G. Schmid, J. Phys. Chem. B, 2001, 105, 1961-1970.

19 C. Maurizio, E. Trave, G. Perotto, V. Bello, D. Pasqualini, P. Mazzoldi, G. Battaglin, T. Cesca, C. Scian and G. Mattei, Phys. Rev. B: Condens. Matter, 2011, 83, 195430.

20 L. D. Menard, H. Xu, S.-P. Gao, R. D. Twesten, A. S. Harper, Y. Song, G. Wang, A. D. Douglas, J. C. Yang, A. I. Frenkel, R. W. Murray and R. G. Nuzzo, J. Phys. Chem. B, 2006, 110, 14564-14573.

21 A. L. Ankudinov, J. J. Rehr, J. J. Low and S. R. Bare, J. Chem. Phys., 2002, 116, 1911-1919.

22 A. I. Frenkel, C. W. Hills and R. G. Nuzzo, J. Phys. Chem. B, 2001, 105, 12689-12703.

23 E. Trave, G. Mattei, P. Mazzoldi, G. Pellegrini, C. Scian, C. Maurizio and G. Battaglin, Appl. Phys. Lett., 2006, 89, 151121.
24 O. V. Safonova, M. Tromp, J. A. van Bokhoven, F. M. F. de Groot, J. Evans and P. Glatzel, J. Phys. Chem. B, 2006, 110, 16162-16164.

25 M. Krause and J. Oliver, J. Phys. Chem. Ref. Data, 1979, 8, 329.

26 K. Hämäläinen, D. P. Siddons, J. B. Hastings and L. E. Berman, Phys. Rev. Lett., 1991, 67, 2850-2853.

27 J. van Bokhoven, C. Louis, J. Miller, M. Tromp, O. Safonova and P. Glatzel, Angew. Chem., Int. Ed., 2006, 45, 46514654.

28 O. Bunau and Y. Joly, J. Phys.: Condens. Matter, 2009, 21, 345501.

29 A. Ankudinov, B. Ravel, J. Rehr and M. Newville, FEFFFIT manual within the FEFF project, University of Washington, Seattle, USA, 1992-1999.

30 A. Das, T. Li, K. Nobusada, Q. Zeng, N. L. Rosi and R. Jin, J. Am. Chem. Soc., 2012, 134, 20286-20289.

31 J. Miller, A. Kropf, Y. Zha, J. Regalbuto, L. Delannoy, C. Louis, E. Bus and J. van Bokhoven, J. Catal., 2006, 240, 222-234.

32 H.-G. Fritsche and R. Benfield, Z. Phys. D: At., Mol. Clusters, 1993, 26, 15-17.

33 In this last case, the thickness of the empty shell defined in the previous section $(\Delta R=1.6 \AA)$ corresponds to a minimum $\mathrm{Au}-\mathrm{O}$ interatomic distance of about $2.5 \AA$ and to an average coordination number for the oxygen shell less than one (within $2.8 \AA$ distance).

34 J. L. C. Fajín, M. N. D. S. Cordeiro and J. R. B. Gomes, J. Phys. Chem. C, 2007, 111, 17311-17321.

35 Z.-P. Liu, P. Hu and A. Alavi, J. Am. Chem. Soc., 2002, 124, 14770-14779.

36 Y. Xu and M. Mavrikakis, J. Phys. Chem. B, 2003, 107, 92989307.

37 A. I. Frenkel, S. Nemzer, I. Pister, L. Soussan, T. Harris, Y. Sun and M. H. Rafailovich, J. Chem. Phys., 2005, 123, 184701.

38 M. A. MacDonald, D. M. Chevrier, P. Zhang, H. Qian and R. Jin, J. Phys. Chem. C, 2011, 115, 15282-15287.

39 D. R. Collins, D. K. Schroder and C. T. Sah, Appl. Phys. Lett., 1966, 8, 323-325.

40 A. Miotello, G. De Marchi, G. Mattei, P. Mazzoldi and C. Sada, Phys. Rev. B: Condens. Matter, 2001, 63, 075409.

41 T. Comaschi, A. Balerna and S. Mobilio, Phys. Rev. B: Condens. Matter, 2008, 77, 075432.

42 Higher disorder in principle could be generated by a monodisperse population of clusters with high configurational disorder but also by a polydisperse population of clusters (in particular, in this range of sizes the interatomic distance strongly depends on the cluster size). Anyway, the dispersion of the cluster size distribution alone cannot be responsible for this high DW value. In fact, we have calculated that even in the worst unrealistic case of a cluster distribution in which the $\mathrm{Au}-\mathrm{Au}$ distances are all equiprobable in the wide range 2.61-2.74 $\AA$, the increase of the effective DW factor would be only $1 / 3$ of what experimentally observed for the sample Au0.5N600. 
43 T. Cesca, C. Maurizio, B. Kalinic, G. Perotto, P. Mazzoldi, E. Trave, G. Battaglin and G. Mattei, Opt. Express, 2012, 20, 16639.

44 H. Imai, K. Arai, H. Imagawa, H. Hosono and Y. Abe, Phys. Rev. B: Condens. Matter, 1988, 38, 12772-12775.
45 G. Wang, T. Huang, R. W. Murray, L. Menard and R. G. Nuzzo, J. Am. Chem. Soc., 2005, 127, 812813.

46 A. Ankudinov, B. Ravel, J. J. Rehr and S. D. Conradson, Phys. Rev. B: Condens. Matter, 1998, 58, 7565. 